\title{
Verzeichnis der Darstellungen
}

Darst. 2- 1: Der Betrieb im Wirtschaftskreislauf

Darst. 2- 2: dto. mit Geldstrom

Darst. 2- 3: Das „Umsystem“ des Betriebes

Darst. 2- 4: Der innerbetriebliche Kreislauf (nach Mellerowicz 1970, S. 7)

Darst. 2- 5: Input-Output-Darstellung des Betriebes

Darst. 2- 6: (Quer- und Längsschnitt-)Funktionen des Betriebes

Darst. 3- 1: $\quad$ Stadien des Entscheidungsprozesses

Darst. 3- 2: $\quad$ Systematik der Prognosemethoden

Darst. 4- 1: Berechnung spezifischer (engpaßbezogener) Deckungsbeiträge

Darst. 4- 2: $\quad$ Simplex-Ausgangs-Tableau (Beispiel)

Darst. 4- 3: $\quad$ Simplex-End-Tableau (Beispiel)

Darst. 4- 4: alternative Substitution

Darst. 4- 5: periphere totale Substitution

Darst. 4- 6: Verbrauchsfunktion (Beispiel Benzinverbrauch)

Darst. 4- 7: Vierphasenschema der Ertragskurven (nach WÖHE 1986, S. 484f.)

Darst. 4- 8: linear-limitationale Faktoreinsatzfunktion

Darst. 4- 9: $\quad$ Faktoreinsatz und Intensität

Darst. 4-10: Faktoreinsatzfunktion bei konstanter Intensität

Darst. 4-11: Gozinto-Graph (Beispiel - nach HorTsch 1985, S. 165)

Darst. 4-12: Direktbedarfsmatrix (Beispiel - ebenda, S. 166)

Darst. 4-13: Die Gesamtkosten als Umkehrfunktion

Darst. 4-14: Kostenverläufe in Abhängigkeit von der Ausbringung

Darst. 4-15: Vierphasenschema der Kostenkurven (nach WÖHE 1986, S. 493f.)

Darst. 4-16: Kostenverlauf bei zeitlicher Anpassung (nach WöHE 1986, S. 511)

Darst. 4-17: Prozeßzeiten von Aufträgen an Maschinen (Beispiel)

Darst. 4-18: Maschinenbelegungspläne des Beispiels (nach ReICHWALD/ MroseK 1985, S. 489)

Darst. 4-19: Arbeitsgänge (Beispiel für eine Strukturanalyse - nach HorTscH 1985, S. 261)

Darst. 4-20: MPM-Netzplan (Beispiel - ebenda, S. 265)

Darst. 4-21: PPS, CAD/CAM und CIM

Darst. 5- 1: $\quad$ Arten der Befragung

Darst. 5- 2: Einteilung der Fragen nach der Antwortmöglichkeit

Darst. 5- 3: $\quad$ Arten der Beobachtung

Darst. 5- 4: Datenmatrix

Darst. 5- 5: Arten der Marketingstrategien 
Darst. 5- 6: Strategieprofil des eigenen Unternehmens im Vergleich zu einem möglichen Wettbewerber (Modellbeispiel - nach BECKER 1988, S. 294)

Darst. 5- 7: $\quad$ Produkt-Markt-Matrix

Darst. 5- 8: Marktwachstum-Marktanteil-Matrix

Darst. 5- 9: Massenmarkt- und Marktsegmentierungsstrategie

Darst. 5-10: Marktsegmentierung nach einem bzw. zwei soziodemographischen

Merkmalen

Darst. 5-11: Marktsegmentierung nach drei soziodemographischen Merkmalen

Darst. 5-12: Quellen der (Produkt-)Ideengewinnung

Darst. 5-13: Checklist für die Auswahl von Produktideen (nach Hütrel 1988)

Darst. 5-14: Gewinnschwelle (Break-even-Punkt)

Darst. 5-15: Preis-Absatz-Funktion

Darst. 5-16: vollkommen elastische bzw. unelastische Nachfrage

Darst. 5-17: Marktformen

Darst. 5-18: Gewinnmaximum bei vollständiger Konkurrenz (nach WöHE 1986, S. 537f.)

Darst. 5-19: horizontale Preisdifferenzierung

Darst. 5-20: Vorteilhaftigkeitsvergleich Reisender/Vertreter

Darst. 6- 1: a) Verlauf des Lagerbestandes bei gleichmäBigem Absatz/ Verbrauch

Darst. 6- 2: $\quad$ Verlauf bei $(\mathrm{s}, \mathrm{x})$-Politik

Darst. 6- 3: Verlauf bei (s,S)-Politik

Darst. 7- 1: Marktwachstum und Marktsättigung (4-Phasen-Schema des Produktlebenszyklus)

Darst. 7- 2: Innovations-Management

Darst. 7- 3: erweitertes bzw. integriertes Produktlebenszyklus Konzept

Darst. 7- 4: (9-Felder-)Markt-Portfolio

Darst. 7- 5: (dto.) Technologie-Portfolio

Darst. 8- 1: Nutzwertanalyse bei der Standortwahl (Beispiel - nach STEINER 1989, S. 118)

Darst. 8- 2: $\quad$ Standort-Portfolio

Darst. 9- 1: Rechtsformen privater Betriebe

Darst. 9- 2: Größenklassen der Kapitalgesellschaften

Darst. 9- 3: vergleichende Zusammenstellung einiger wichtiger Rechtsformen

Darst. 9- 4: Kooperation, Konzentration und Wachstum

Darst. 9- 5: Formen von Unternehmenszusammenschlüssen

Darst. 9- 6: Konzern- und abhängiges Unternehmen

Darst. 9- 7: Formen öffentlicher Betriebe 
Darst. 10- 1: Organisation und Umwelt

Darst. 10- 2: organisationswissenschaftliche Ansätze (nach Hill/FeнLвaum/ ULRICH 1981)

Darst. 10- 3: Formen von Leitungssystemen

Darst. 10- 4: Formen der Aufbauorganisation

Darst. 10- 5: funktionale Organisation

Darst. 10- 6: divisionale Organisation

Darst. 10- 7: regionale Organisation

Darst. 10- 8: Matrix-Organisation

Darst. 10- 9: Produkt-Management als Mischform

Darst. 11- 1: Einordnung von „Management by-Techniken“ (nach Puluig 1980, S. 59)

Darst. 11- 2: „Regelkreis“ der Strategischen Unternehmensführung

Darst. 11- 3: Arten der Mitbestimmung

Darst. 12- 1: Funktionendiagramm von Stellen in der Personalabteilung (nach OeCHSLER 1988, S. 35)

Darst. 12- 2: Personalauswahl-Verfahren (nach STOPP 1989, S. 58)

Darst. 12- 3: Verfahren der Arbeitsbewertung

Darst. 12- 4: Beispiel des Rangreihenverfahrens der analytischen Arbeitsplatzbewertung

Darst. 12- 5: Arten des Prämienlohns

Darst. 12- 6: Rowan- und Halsey-Prämienlohnsystem (Beispiel)

Darst. IVE-1: Geld- und Güterströme aus der Sicht des Betriebes

Darst. IVE-2: Abgrenzung „Einzahlungen“/"Einnahmen“ (nach WÖHE 1986, S. 875)

Darst. IVE-3: Betriebliche Zahlungsströme

Darst. 13- 1: Einteilung der Investitionen nach dem Anlaß

Darst. 13- 2: Einteilung der Investitionen nach dem Objekt

Darst. 13- 3: Beispiel-Grundmodell

Darst. 13- 4: vollständiger Finanzplan (Beispiel)

Darst. 13- 5: vollständiger Finanzplan (Beispiel: Zinsänderung)

Darst. 13- 6: vollständiger Finanzplan (Beispiel: Entnahmen)

Darst. 13- 7: Verfahren der Investitionsrechnung bei Einzelobjekt-Entscheidungen

Darst. 13- 8: kritische Menge (zweier Investitionsobjekte)

Darst. 13- 9: Gewinnschwellebeiderpay-off-Methode(nachKAPPLER/REHKUGLER 1985, S. 801)

Darst. 13-10: dynamische Verfahren (Alternativ-Einteilung)

Darst. 13-11: Berechnung der abgezinsten Einzahlungen (Beispiel)

Darst. 13-12: Berechnung der abgezinsten Einzahlungen (korrigiert)

Darst. 13-13: Berechnung von Varianten (Beispiel)

Darst. 13-14: Kapitalwertfunktion, in Abhängigkeit von der Nutzungsdauer (nach KapPLER/RehKugleR 1985, S. 821) 
Darst. 14- 1: Zahlungsströme und Kapital-/Geld-/Finanzbedarf (nach ReHKuGLER/SCHINDEL 1989b, S. 34)

Darst. 14- 2: Arten der Finanzierung

Darst. 14- 3: Kapazitätserweiterungseffekt (Beispiel)

Darst. 14- 4: Arten der Aktien

Darst. 14- 5: Arten des Leasing

Darst. 14- 6: Arten des Factoring

Darst. 14- 7: Leverage-Effekt (positiv - Beispiel)

Darst. VE- 1: Abgrenzung „Einnahmen“/"Erträge“ (nach WöHE 1986, S. 879)

Darst. VE- 2: Verhältnis von 8 Grundbegriffen

Darst. 15- 1: Teile des Jahresabschlusses (und Lagebericht)

Darst. 15- 2: Die Gegenüberstellung von „Vermögen“ und „Kapital“ in der Bilanz

Darst. 15- 3: Schema eines aktiven Bestandskontos

Darst. 15- 4: Schema des IKR 1986

Darst. 15- 5: Grundsätze ordnungsmäßiger Buchführung bzw. Bilanzierung (nach Heinhold 1988, S. 47)

Darst. 15- 6: Bilanz-Mindest-Gliederung

Darst. 15- 7: Arten der Zeitabschreibung

Darst. 15- 8: verschiedene Abschreibungsarten (Beispiel)

Darst. 15- 9: Abgrenzung „Aufwand“/,,Kosten“"

Darst. 15-10: Beständedifferenz- und Bewegungsbilanz

Darst. 15-11: GuV-Gliederungsschema nach $\$ 275$, Abs. 2 und 3 HGB (für große Kapitalgesellschaften)

Darst. 15-12: Bilanz-Gliederungsschema nach $\S 266$, Abs. 2 HGB (für mittlere und große Kapitalgesellschaften)

Darst. 16- 1: betriebliche Aufwendungen (Kl. 6 des IKR 1986)

Darst. 16- 2: Betriebsrechnungsbogen (schematisch)

Darst. 16- 3: (differenzierende) Zuschlagskalkulation, schematisch

Darst. AI- 1: Einteilung der Steuern

Darst. AI- 2: Das „Steuersystem“

Darst. AII-1: Input-Output-Darstellung der EDV

Darst. AII-2: Grundaufbau der EDV

Darst. AII-3: Möglichkeiten der Dateneingabe

Darst. AII-4: Arten von Druckern

Darst. AII-5: Einteilung der System-Software

Darst. AII-6: Einteilung der Programmiersprachen

Darst. AII-7: Einteilung der Standard-Software

Darst. AII-8: (logische) Struktur der Datenorganisation

Darst. L- 1: Verlauf bei $(t, S)$-Politik

Darst. L- 2: Matrix-Organisation im Absatzbereich

Darst. L- 3: $\quad$ Lohnzuordnung zu Arbeitswerten (Beispiel)

Darst. L- 4: vollständiger Finanzplan (Beispiel: Alternative)

Darst. L- 5: Leverage-Effekt (negativ - Beispiel) 
